\title{
CONTROLANDO LOS RESULTADOS DEL SISTEMA PENAL SOBRE EL DERECHO ADMINISTRATIVO DEL DERECHO PENAL
}

\author{
CONTROLLING THE GENERAL OUTPUT OF THE PENAL SYSTEM \\ ON ADMINISTRATIVE LAW OF CRIMINAL LAW
}

\author{
JAVIER WILENMANN ${ }^{*}$
}

\begin{abstract}
RESUMEN: El artículo analiza las dificultades que tiene el sistema penal en controlar sus resultados agregados. En él, se muestra cómo la conjunción de una cultura dominante fijada en procesos de toma de decisión individual y un diseño institucional que no solo impone el mismo tiempo de orientación, sino que disgrega las decisiones relevantes entre agentes no coordinados, tiende a producir ese efecto. El artículo analiza finalmente las alternativas administrativas de imposición de control por los resultados agregados que respetan, sin embargo, el tipo de proceso de toma de decisión que caracteriza al derecho penal.
\end{abstract}

Palabras clave: sistema penal, salidas anticipadas, libertad condicional, penas sustitutivas, determinación de la pena, derecho administrativo, organismos reguladores

SUMMARY: The article analyzes the difficulties that the Chilean penal system shows in controlling its outcomes. It shows that the combination of a legal culture fixed with individual judgements and an institutional design that maintains that focus and disperses decisionmaking powers between non-coordinated actors, might tend to produce such an effect. The article offers a reconstruction of the administrative alternatives of control of penal outcomes that can be respectful of the type of decision-making system that makes out criminal law.

Key words: criminal justice system, parole, probation, administrative law, regulatory agencies.

\section{INTRODUCCIÓN}

La cultura de expertos y actores en el ámbito del derecho penal, unida a la configuración del sistema de toma de decisiones que informa, contribuye a la producción de resultados agregados desordenados.

En el análisis del sistema penal, los abogados y, en particular, los penalistas y procesal-penalistas afirman propiedad. Desde que se formaliza en el procedimiento, el ámbito institucional penal está densamente asociado a la cultura interna de estos grupos ${ }^{1}$. La cultura legal-penal no solo configura, como es natural, la acción que se desarrolla dentro de esa práctica -los abogados y jueces hablan el lenguaje del derecho-, sino que también tienden a asumir la posición de opinión política experta a su respecto².

* Doctor en Derecho, Albert-Ludwigs Universität Freiburg, Profesor Asociado, Facultad de Derecho Universidad Adolfo Ibáñez. Dirección postal: Diagonal Las Torres 2640, Peñalolén, Santiago. Dirección electrónica: javier.wilenmann@uai.cl

1 El concepto proviene de Friedman (1975) cap. 9.

2 En general, al menos tres comunidades de conocimiento reclaman expertise en materia penal: los penalistas, los diseñadores del sistema procesal penal y los expertos en seguridad pública. Véase Hathazy (2016); PALACiOS 
WilenMann, Javier " "Controlando los resultados del sistema penal sobre el derecho administrativo del derecho penal"

Esa cultura que clama propiedad sobre "lo penal" se concentra en procesos de toma de decisión respecto de individuos y no en la producción de resultados agregados ${ }^{3}$.

En efecto, el derecho penal establece las condiciones en las que el derecho autoriza (o manda) a aplicar una sanción penal a un individuo. Los penalistas se preocupan de reconstruir en detalle los procedimientos argumentativos que debieran controlar ese proceso individual. Tanto por la estructura misma de esa cultura, como por importaciones de traumas foráneos, el control del sistema como un todo no solo está fuera del ámbito de sus preocupaciones, sino que puede ser incluso rechazado como una especie de empresa anti-libertaria.

Los procesalistas, por su parte, han asumido una fijación centrada en las garantías del individuo y en la legitimación procedimental de la pena ${ }^{4}$. ¿Cómo se configura un contexto institucional en el que no se produzcan malos tratos al imputado y en el que los jueces se orienten exclusivamente a la determinación, ordenada por reglas, de la culpabilidad o inocencia del imputado? ¿Cómo se reducen al máximo razonable los riesgos de falsos positivos?

Por cierto, las transacciones que llevaron a la reforma procesal penal implicaron la instauración de preocupaciones burocráticas de eficiencia ("gerencialismo") 5 . Por ello, a su respecto también se discute sobre mecanismos de gestión. Pero el alcance de los mecanismos es limitado tanto dentro de la extensión del sistema penal -sigue en lo esencial al procedimiento controlado por abogados - como en sus pretensiones- optimizar el uso de los espacios procedimentales y proveer de mecanismos que agilicen la obtención de condenas o el abandono de casos ${ }^{6}$. La inserción del procedimiento dentro del funcionamiento total de un sistema, es algo que no es en general considerado por los procesalistas ${ }^{7}$.

Visto desde fuera, esta dinámica genera un sistema sin preocupación por los resultados agregados que produce. Y ello, en condiciones que buena parte de los problemas del sistema penal solo pueden ser entendidos asumiendo una perspectiva global. Concentrándose en los resultados - lo que aquí nos interesa resaltar- esto es sencillo de advertir. El uso excesivo de la cárcel, por ejemplo, no solo tiene efectos innecesarios o excesivos en los mismos condenados, sino que priva de posibilidades de sustento a sus familias, disminuye la eficacia en la generación del control de los niños en procesos de socialización ${ }^{8}$, desorganiza la economía de ciertas zonas y, con ello, puede tender a aumentar los niveles de delincuencia en vez de disminuirlos 9 . Y como todo el financiamiento del sistema penal es público, ello implica no solo que los recursos públicos se gastan de un modo ineficiente frente a

(2011). Aunque, por lo mismo, el campo en cuestión puede ser visto como lugar de conflicto, la cultura de al menos dos grupos comparte la configuración legal de fijación individual en cuestión.

3 El diagnóstico ya se encuentra en ZiMring y HaWkins (1993) pp. i y siguientes.

4 "El procedimiento penal implica (...) la asunción de dos cuestiones: primero, la producción de una decisión definitiva (...) y, segundo, que la misma genere un efecto vinculante como consecuencia de su legitimación formal (...)." Horvitz y López (2002) p. 25.

5 Véase recientemente GonZÁlEz (2018) cap. 2.

${ }^{6}$ Esto es tradicionalmente visto como una herencia de la fijación europeo-continental con la idea de legalidad (=a todo delito debe seguir una respuesta estatal) y, con ello, a la conceptualización de todo caso que se desvíe de esa pretensión como excepción de oportunidad. Véase Duce (2002) pp. 248-52; Horvitz y López (2002) pp. 448-50.

7 STUnTz (1997) pp. 1-5.

8 Para una sencilla referencia a la literatura empírica sobre el punto, véase EWALD y UGGEN (2012) pp. 92s.

9 Hagan y Dinovitzer (1999) pp. 122s. 
sus alternativas -gastar más en servicios sociales seguramente tendría mejores efectos en la disminución de la delincuencia-, sino que se usan a veces de modo derechamente contraproducente. La falta de control de cuidado en el mantenimiento de la correlación entre capacidad carcelaria y uso de la cárcel genera hacinamiento, produciendo no solo espacios más insalubres y violentos de vida, sino también un rendimiento todavía peor de la cárcel en la disuasión individual futura de cada condenado ${ }^{10}$.

Pese a todo lo anterior, el diseño institucional del sistema penal -y la cultura que lo impregna- no tiene una orientación al control de esos resultados. No hay mecanismos u organismos que controlen que los resultados se ajusten a ciertos parámetros (por ejemplo: que la tasa de encarcelamiento se ubique dentro de ciertos márgenes), que los recursos escasos del sistema se utilicen donde tienen más rendimiento, o que los recursos que se le asignan al sistema de justicia penal tengan un uso comparativamente mejor que la asignación a otros sistemas que pretenden producir resultados en el mismo ámbito (asistencia social, por ejemplo). Aquello que es (o debiera ser) normal en la producción de políticas públicas y en el funcionamiento de la administración en otros ámbitos ${ }^{11}$, en el sistema de justicia penal se encuentra fuera de consideración.

El más claro ejemplo de esta dinámica se encuentra en los resultados que siguieron a la implementación de la reforma procesal penal. Contra lo que esperaban los agentes de la reforma ${ }^{12}$, su efecto fue un aumento masivo de la tasa de encarcelamiento ${ }^{13}$. Programado para aumentar la eficacia en la aplicación de sanciones, el sistema no sabía controlar sus propios resultados ${ }^{14}$. Y ese efecto negativo continuó produciéndose hasta que el incendio de la cárcel San Miguel generó un escenario político favorable a la utilización de medidas ad hoc de reducción de la población carcelaria. Incluso después de esos ajustes, el sistema sigue comportándose de modo volátil.

Este artículo pretende comenzar a ofrecer una salida a esta situación. Su objetivo es dar cuenta del hecho de que el derecho penal no es solo una definición de las condiciones de punibilidad de individuos, sino también un sistema de reglas de orientación y control de una burocracia masiva cuya acción tiene un enorme impacto social ${ }^{15}$. Por decirlo en términos que pueden resultar heréticos en el ámbito penal: el derecho penal no es solo un "derecho del ciudadano"; sino que es ante todo un programa de regulación de una máquina burocrática ${ }^{16}$. Como tal, se trata de un ámbito legal que también debe ser pensado en lógica administrativa ${ }^{17}$. Eso no quiere decir, por cierto, que consideraciones administrativas de eficacia deban borrar el tipo de procedimientos argumentativos que caracterizan a la toma de decisiones en materia penal. Antes, un buen diseño administrativo necesita asumir

\footnotetext{
10 Sobre los efectos individuales del hacinamiento, véase solo HanEY (2012) pp. $593 \mathrm{~s}$.

11 El punto es enfatizado por BARKOW (2017).

12 DeShazo y Vargas (2006); Riego (2000).

13 Salinero (2012).

14 El punto, referido a Brasil, es en parte desarrollado por FonseCA (2018).

15 En la tradición de teoría penal, el punto también es enfatizado por ChIAO (2017), EL MISMO (2018) cap. 1.

16 Barkow (2014); Dolovich y Natapoff (2017).

17 El argumento, por cierto, no es nuevo en la literatura comparada. Se encuentra en la base del libro de ZIMRING y HaWKInS (1993). Y el punto ha sido llevado en buena medida al extremo por BARKOW (2017) pp. 42-44.
} 
que el sistema que debe controlar se encuentra programado de un modo que supone preeminencia de la atribución de culpabilidad - un proceso de toma de decisión naturalmente individual. Como veremos, la complejidad del diseño administrativo se encuentra determinada precisamente por ello. Ello no quiere decir, sin embargo, que la racionalidad administrativa deba ser abandonada ${ }^{18}$.

Enfocado en un solo punto -el control de los resultados agregados de las sancionesel artículo ofrece una reconstrucción de la relevancia de pensar también al derecho del sistema penal como un proyecto de regulación de una burocracia ${ }^{19}$.

Para ello, en primer lugar, daré cuenta, de dos modos alternativos en que un sistema puede controlar sus resultados: de modo centralizado (orgánico) y de modo descentralizado (inorgánico). Aunque, como veremos, hay factores que pueden incidir en la generación de un control espontáneo, los datos muestran que los sistemas latinoamericanos, incluyendo el chileno, no tiene esa capacidad. En segundo lugar, daré cuenta de las razones estructurales formales que influyen en esa incapacidad de generar coordinación. Finalmente, en una tercera parte, daré cuenta de los modelos comparados existentes para la producción de ese control de un modo centralizado y orgánico.

\section{LOS MECANISMOS DE PRODUCCIÓN DEL RESULTADO}

\subsection{Mecanismos informales de CONTROL Y la tesis de la eSTABilidad Del CASTigo}

Aunque volveremos sobre el punto más adelante, asúmase para efectos del argumento que el sistema penal (i) se configura en torno a una cultura orientada a la toma de decisiones en el caso individual y (ii) que no hay coordinación en la toma de esas decisiones que permita contrarrestar la tendencia de un sistema de esa clase a la volatilidad de sus resultados. Un sistema de esta clase es, en ese sentido, un sistema descoordinado.

La configuración descoordinada del sistema penal sugiere que, de dejarse a la pura reproducción siguiendo la lógica individual que predomina en el proceso, los resultados van a ser arbitrarios y, probablemente, volátiles.

Llegar a esa conclusión sin más puede ser, sin embargo, apresurado. En particular, no es inusual que, pese a la falta de coordinación, un sistema institucional estabilice sus resultados sea porque su disponibilidad de recursos se mantiene más o menos inalterada, sea porque cuenta con mecanismos informales que tienden a ese resultado. Si uno, por ejemplo, utiliza las tasas de encarcelamiento como proxy del resultado producido por el sistema -un proxy útil pero limitado- varios sistemas muestran evoluciones más o menos planas de sus resultados. Así, a pesar de las denuncias de expansión masiva del castigo ligado al

\footnotetext{
18 Una exposición temprana llamando a pensar el sistema de justicia penal de este modo, ya al interior de la comunidad legal, se encuentra en ZimRING y HaWkins (1993) p. xii.

19 Es importante notar lo limitado que es el punto. Administrativamente visto, el sistema también debe preocuparse que cada programa (= sanción y medidas dentro de la sanción) produzca los mejores efectos posibles respecto de una gran cantidad de consecuencias buscadas. Ese micro-manejo es parte central de la racionalidad administrativa que debe buscar el sistema. Me concentro aquí solo en resultados agregados porque se trata del aspecto probablemente más descuidado.
} 
sistema neoliberal internacional ${ }^{20}$, buena parte de los países europeos occidentales (con la excepción notable del Reino Unido) han mantenido niveles de castigo a veces inferior y a veces solo ligeramente superior a lo que tenía lugar hace 40 años ${ }^{21}$.

En 1973, y haciendo referencia al caso de los Estados Unidos, Blumstein y Cohen le dieron forma de teoría general a la observación de que la tasa de encarcelamiento se había mantenido dentro de márgenes estrechos por los últimos 50 años $^{22}$. A la vista de las variaciones relevantes en tasas de delincuencia que era posible percibir a la época y, pese a ello, el mantenimiento de los niveles agregados de castigo, ambos postularon que el proceso institucional de castigo tenía características homeostáticas que disponía a una definición del número de acciones efectivamente castigables de modo más o menos constante. Ello porque las sociedades fijarían, en los hechos, ciertas características que constituyen barreras de intolerancia frente a ciertas desviaciones y esas barreras cambiarían en base a una fijación estable de necesidad de castigo. Así, si los niveles de acciones castigables definidas en base a un umbral de tolerancia determinado disminuyen, las fuerzas que empujan los procesos de criminalización (organizaciones de persecución, legislación, etc.) llevarían a ampliar la barrera de tolerancia y constituir a acciones menores en criminales. Si, en cambio, esos niveles aumentaran, esas mismas fuerzas empujarían a aumentar los niveles de tolerancia, disminuyendo el nivel de castigo de acciones que pasarían a ser consideradas menores. Ese postulado teórico, según Blumstein y Cohen, encontraría validación empírica en el hecho de que entre 1930 y 1970, la tasa de encarcelamiento en los Estados Unidos mostraba una variación leve ${ }^{23}$.

La teoría de la estabilidad del castigo fue refutada del modo más espectacular posible $^{24}$. Justo después de la famosa publicación de Blumstein y Cohen, la tasa de encarcelamiento en los Estados Unidos comenzó a aumentar drásticamente. En términos gráficos, ello condujo al "gráfico por antonomasia" en la discusión académica y pública en la actualidad en ese país: una línea más o menos estable de encarcelamiento hasta principios de los años 70 que luego creció casi 7 veces hasta alcanzar su punto más alto a principios de los años $2000^{25}$. Estados Unidos pasó de ser un ejemplo de validación empírica de la estabilidad del castigo al ejemplo por antonomasia de la falta de estabilidad natural del sistema penal.

Lo anterior no implica, sin embargo, que al menos una parte de las asunciones de Blumstein no tuvieran un grano de verdad. Más allá del caso de Estados Unidos (y, en lo que nos interesa a nosotros, de Chile y de Latinoamérica), varios países europeos han mantenido resultados agregados más o menos constantes ${ }^{26}$, sin contar -como algunos Estados

20 Véase, por ejemplo, la crítica de ZEDner (2002) pp. 352 ss. al carácter generalizador del diagnóstico de GARLAND (2002).

21 Para los efectos del argumento véase solo LACEY (2008) pp. 26-29. El caso más notable es, probablemente, Canadá, cuya similitud en la evolución de las tasas de delincuencia con los Estados Unidos podría sugerir variaciones similares en niveles de castigo -algo simplemente errado. Véase Doob Y Webster (2006) pp. 327-32.

22 Véase Blumstein y Cohen (1973). El punto fue retomado en varios artículos del grupo de trabajo de Blumstein, incluso después de que se hiciera evidente la refutación de la tesis. Véase, al respecto, RudDELL y FEAR (2005) pp. 3-5.

23 Blumstein y Cohen (1973).

24 Zimring (2010).

25 Aviram (2017); Pfaff (2008) pp. 547-51.

26 Véanse, por ejemplo, los datos en Ruddell y Fear (2005), pp. 13-16. 
WiLenMann, Javier "Controlando los resultados del sistema penal sobre el derecho administrativo del derecho penal"

de los Estados Unidos- con arreglos institucionales directamente tendientes al control de sus resultados.

En un famoso artículo publicado en 1994, Joachim Savelsberg le atribuyó esa capacidad de controlar sus resultados a un mayor aislamiento por parte de la cultura legal y de la burocracia ligada al sistema de justicia penal, paradigmáticamente presente en Alemania, del sistema político ${ }^{27}$. Si la cultura legal mantiene formas de razonamiento aisladas de presiones políticas, ella determina la acción de la burocracia y los recursos de la burocracia cambian poco, entonces probablemente los resultados se mantengan controlados. Cualquiera sea la opinión que uno tenga de esta tesis, ella muestra que ciertos sistemas muestran capacidad de control informal de sus resultados: la propia interacción de la cultura legal con los actores que gestionan la burocracia puede actuar como mecanismo tendiente a la estabilización. La necesidad de pensar administrativamente el derecho penal es menor en esos casos.

En sistemas, en cambio, que muestran resultados volátiles y tendencia incontrolada al aumento del castigo, la preocupación administrativa es central. Este es el caso de Latinoamérica y, desafortunadamente, de Chile ${ }^{28}$. La Tabla 1 muestra el nivel de volatilidad, con tendencia clara al boom, que ha tenido el uso de la cárcel en Latinoamérica en los últimos tiempos.

TABLA 1

Evolución tasas de encarcelamiento en Latinoamérica (confección propia a partir datos World Prison Brief)

\begin{tabular}{|l|c|r|r|r|r|r|}
\hline \multicolumn{1}{|c|}{ País } & \multicolumn{1}{c|}{1995} & 2000 & \multicolumn{1}{c|}{2004} & \multicolumn{1}{c|}{2010} & 2016 & \multicolumn{1}{c|}{ Variación } \\
\hline Argentina & \multicolumn{1}{c}{13} & 102 & 168 & 157 & $174(2015)$ & $+138 \%$ \\
\hline Brasil & 107 & 133 & 180 & 249 & 347 & $+224 \%$ \\
\hline Bolivia & $69(1996)$ & 95 & 73 & 92 & 142 & $+106 \%$ \\
\hline Chile & 153 & 215 & 226 & 320 & 242 & $+58 \%$ \\
\hline Colombia & 90 & 128 & 159 & 183 & 243 & $+170 \%$ \\
\hline Costa Rica & 100 & 193 & 209 & 259 & $370(2014)$ & $+270 \%$ \\
\hline República Dominicana & 159 & 173 & 140 & 208 & 235 & $+48 \%$ \\
\hline Guatemala & 58 & 62 & 70 & 78 & 125 & $+116 \%$ \\
\hline Ecuador & 85 & 64 & 84 & 79 & 160 & $+88 \%$ \\
\hline El Salvador & 125 & 132 & 201 & 399 & 579 & $+363 \%$ \\
\hline Honduras & 158 & 184 & 160 & 154 & 200 & $+27 \%$ \\
\hline México & 101 & 156 & 186 & 194 & 177 & $+75 \%$ \\
\hline Nicaragua & 98 & 128 & 116 & 113 & 238 & $+143 \%$ \\
\hline Paraguay & 57 & 60 & 106 & 99 & 167 & $+193 \%$ \\
\hline Panamá & 237 & 283 & 345 & 336 & 423 & $+78 \%$ \\
\hline Perú & 87 & 107 & 114 & 155 & 251 & $+189 \%$ \\
\hline Uruguay & 99 & 135 & 207 & 257 & 306 & $+209 \%$ \\
\hline Venezuela & $101(1996)$ & 58 & 76 & 140 & 173 & $+71 \%$ \\
\hline
\end{tabular}

\footnotetext{
27 SAVELSBerg (1994). Un punto similar, respecto de la estabilidad en la determinación de la pena, es efectuado por HÖRNLE (2013).

${ }^{28}$ Ariza e IturRalde (2018); IturRalde (2010); Sozzo (2016).
} 
Como veremos a continuación, la existencia de variaciones violentas en las poblaciones carcelarias implica un desajuste de los factores que mantienen la estabilidad del castigo. Aunque podría tratarse de un efecto buscado -los actores de un sistema podrían buscar aumentar o disminuir el nivel de castigo- la presencia simultánea de hacinamiento carcelario muestra que ello no es así o, en todo caso, el aumento del nivel de castigo también tiene lugar de modo desordenado. Ello hace necesario entender qué produce, al menos formalmente, el efecto de desajuste.

\subsection{El OUTPUT DEL Sistema Y LOS MeCANiSMOS FORMALES QUE LO PRODUCEN}

Para efectos de graficar cómo funciona la producción del resultado agregado "castigo" en un momento determinado, podemos hacer uso del ejemplo paradigmático del encarcelamiento.

Las variaciones en el encarcelamiento pueden representarse como el resultado de un flujo: la población carcelaria en el momento N2 (PCN2) es el resultado de la adición de aquélla presente en N1 (PCN1) y el total de ingresos menos el total de las salidas de la cárcel por parte de condenados entre N1 y N2. El flujo puede ser representado sencillamente como muestra la Figura 1.

FIGURA 1

Flujo carcelario de condenados
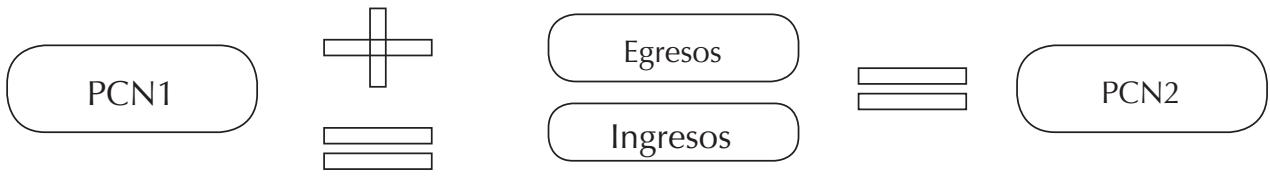

A su vez, los factores "ingresos" y "egresos" son el resultado de distintos actos institucionales.

Los ingresos se corresponden sencillamente con el total de condenas a penas privativas de libertad ejecutadas y el total de sujetos a los que se les ha impuesto prisión preventiva (o detención, si se cuenta aquí) entre N1 y N2.

Aunque estos dos factores pueden parecer sencillos de determinar, ellos en realidad se ven condicionados por una gran cantidad de actos institucionales anteriores. Las condenas, por ejemplo, dependen de una serie de actos institucionales que revisaremos con más detalle más adelante. Ejemplo: toda salida alternativa, todo principio de oportunidad o facultad de no perseverar, etc., filtra del número total de condenas que podría producirse en un momento determinado respecto una demanda nominal mayor. Al mismo tiempo, toda aplicación de una pena que no sea efectivamente privativa de libertad -incluyendo a las penas sustitutivas- produce el mismo efecto si el resultado que se quiere controlar es el encarcelamiento.

Los egresos corresponden, a su vez, al total de actos institucionales cuyo efecto es el término de la causa de privación de libertad respectiva. Tratándose de condenados, el cumplimiento (con o sin abono de condena), la libertad condicional y el indulto, más allá de la muerte del condenado, son los casos más relevantes de actos institucionales que conducen 
al resultado "egreso". De estas posibilidades de salida, varias de ellas se ven determinadas al menos parcialmente por actos institucionales anteriores. El cumplimiento o el tiempo mínimo para solicitar libertad condicional, por ejemplo, dependen de la cuantía de la pena fijada al momento de su determinación.

Como es obvio, la tasa de encarcelamiento tiende a mantenerse estable en la medida en que los ingresos y los egresos se ubiquen en parámetros similares. Ella disminuye en la medida en que los egresos superen a los ingresos, y aumenta en la medida en que los ingresos superen a los egresos. Al revés, la verificación de que la tasa varía muestra que los factores de la ecuación no se han mantenido alineados. Sea por inestabilidad interna de la burocracia -su eficacia aumenta y disminuye sin mucho control-, sea por aumento de recursos que le permite procesar más casos, o sea por variaciones en los marcos institucionales que permiten el procesamiento, variaciones en las tasas de encarcelamiento muestran un sistema desalineado en el manejo de la ecuación.

Cuando el sistema se encuentra desalineado, es improbable que se produzcan ajustes espontáneos que tiendan a alinearlo. Los factores que influyen en la producción del resultado definitivo son demasiado heterogéneos y complejos para que ello tenga lugar. En el próximo apartado intentaré explicar las razones por las que ello es así. Para ello, daré cuenta en primer lugar de las características orgánicas centrales del sistema. Posteriormente explicaré las razones por las que, en esas condiciones, conseguir coordinación espontánea para un resultado determinado (ejemplo: alinear) es improbable.

\section{EL FUNCIONAMIENTO MACRO-ESTRUCTURAL DEL SISTEMA PENAL Y SU RESULTADO}

\subsection{El SISTEMA PENAL Y SU CARACTERÍSTICA ORGÁNICA CENTRAL: LA FRAGMENTACIÓN EN}

\section{LA TOMA DE DECISIONES}

El sistema de justicia penal se corresponde con lo que en teoría institucional es conocido como un campo organizacional. Un campo se ve constituido por el conjunto de interacciones en un ámbito de acción institucional reconocible como tal ${ }^{29}$. Aunque, por ejemplo, en materia penal decenas de miles de personas realizan distintas acciones de persecución, defensa, juicio, etc., en todos ellos podemos entender culturalmente que se está desarrollando un tipo de proceso al que llamamos "persecución penal” o alguna definición similar.

Un campo admite tres niveles de observación en su descripción: el nivel individual, el nivel de organizaciones individuales, o el nivel de poblaciones de organizaciones (el nivel de campo).

Así, por ejemplo, uno puede describir el sistema de justicia penal como un campo de interacciones de jueces, fiscales, abogados, imputados y policías individuales. Esa imagen es la que tiende a asumir la literatura jurídica. Si desarrollan correctamente su función, todos esos individuos son estructuralmente equivalentes entre sí, porque realizan acciones definidas por la misma lógica de acción por una organización. Ello permite aumentar el nivel de

29 DiMaggio y Powell (1983). 
generalidad en la descripción, sin demasiada pérdida de precisión, y referirse a organizaciones individuales. Por ejemplo, uno puede describir lo que tiene lugar en el Cuarto Juzgado de Garantía de Santiago haciendo referencias a las acciones de la fiscalía local de Nuñoa, de ciertos cuerpos específicos de Carabineros, etc. De nuevo, como la organización institucional provee que distintas unidades organizacionales sean estructuralmente equivalentes entre $s^{30}{ }^{30}$, uno puede aumentar todavía más el nivel de generalidad de la descripción y pasar a describir el "campo" total del sistema como interacciones de "poblaciones" de organizaciones. En el sistema de justicia penal, visto como campo, las poblaciones de organizaciones designadas como "policía”, "Ministerio Público", "Defensoría Penal Pública” (= el conjunto de todas las organizaciones individuales que asumen esa identidad), etc., interactúan para producir el resultado global de que se trata. En lo que nos interesa a nosotros, la asunción del punto de vista del campo institucional es fundamental.

Más allá de referirse a un campo, en su consideración espacial, el sistema de justicia penal también se corresponde dinámicamente con un proceso. En la imagen ya clásica legada por el reporte The Challenge of Crime in a Free Society ${ }^{31}$ ese proceso puede ser descrito como un flujo en el que ciertas fuentes de input (los delitos) conocidos por la policía se ramifica en sus resultados, dependiendo de combinaciones de distintos factores relevantes a estos efectos (presencia o ausencia de un arresto, entidad del delito, prueba, etc.) que son procesados, a veces de modo cooperativo, otras de modo adversarial, por las distintas poblaciones organizacionales comprometidas.

Para nuestros efectos, no es necesario contar con una descripción de flujo que pretenda ser exhaustiva. Más bien, la Figura 2 permite describir simplificadamente el proceso conducente al resultado "sanción".

\section{FIGURA 2}

El proceso de producción del resultado penal global

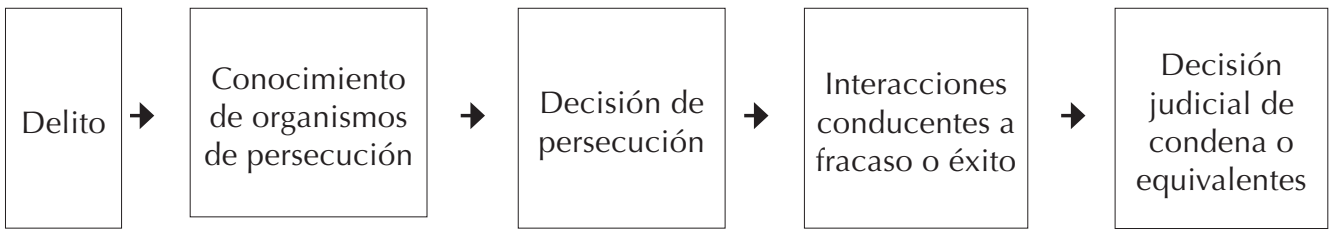

Un diagrama sencillo de flujo como la Figura 2 permite describir el conjunto de interacciones que conducen a la producción individual "sanción penal (o equivalente) 32 " -el diagrama asume así el nivel de descripción puramente individual.

Pero el proceso en cuestión también puede ser transformado a nivel de campo simplemente agregando todos los procesos que se desarrollan en el sistema. El resultado

30 Dimaggio y Powell (1983) p. 148.

31 President's Commission of Law Enforcement and Administration of Justice (1967) pp. 8-9.

32 Hago referencia a "equivalentes" por el hecho de que, formalmente, varios modos de acción que tienen peso coactivo y pueden implicar gasto de recursos públicos, no son considerados como tales. El caso obvio es la suspensión condicional del procedimiento. 
de ese conjunto de procesos genera el output total de sanciones. Ello todavía no permite cuantificar el efecto que el sistema tiene en los recursos públicos (impacto fiscal) o en sus destinatarios (impacto social), ya que cada sanción tiene por naturaleza y duración efectos diferenciables y el efecto específico de la sanción puede cambiar por decisiones posteriores (indulto, libertad condicional). Pero la Figura 2 sirve como contraste (individual) para explicar las razones por las que a nivel agregado el sistema no tiene coordinación ${ }^{33}$.

En efecto, aunque la Figura 2 parece describir procesos ordenados, hay varias razones por las que, considerado a nivel del resultado global que produce, el sistema no funciona así.

En primer lugar, el resultado final depende de acciones que las distintas organizaciones que participan del campo toman de modo contingente o necesariamente autónomo. Ejemplo: el Ministerio Público no puede coordinar, en el caso individual, su actuación con los tribunales. Y, es probable, que además las acciones de la policía conducentes a uno u otro resultado no estén plenamente coordinadas con el Ministerio Público. Aunque visto a nivel individual algunos casos de no-coordinación pueden ser vistos como necesidad del sistema, en relación con el resultado final producido ello genera problemas de control.

La Figura 3 intenta graficar la dispersión de tomadores de decisión que conduce al resultado agregado.

\footnotetext{
33 En la literatura reciente, de hecho, el punto tiende a utilizarse para oponerse incluso al uso del concepto de "sistema" al hablar de la persecución penal a nivel de campo: su falta de coordinación a nivel de diseño (y en algunos países también en su funcionamiento informal) haría que la idea de sistema solo confunda. Véase MAYEUX (2018).
} 
FIGURA 3

Tomadores de decisión en la generación del output del sistema de justicia pena

\begin{tabular}{|c|c|c|c|}
\hline \multicolumn{2}{|c|}{$\begin{array}{l}\text { Co-legisladores } \\
\text { Criminalización; cuantía } \\
\text { nominal penas; criterios } \\
\text { de fijación de pena real }\end{array}$} & \multicolumn{2}{|c|}{$\begin{array}{l}\text { Orientación decisión: política. } \\
\text { Práctica chilena: Sin fijación en costos } \\
\text { fiscales en materia penal. } \\
\text { Efectos presupuestarios: sistémico-generales }\end{array}$} \\
\hline $\begin{array}{l}\text { Fiscales: decisión } \\
\text { de persecución; } \\
\text { decisión de tipo de } \\
\text { sanción a imponer } \\
\text { (salidos alternativas) }\end{array}$ & \multicolumn{3}{|c|}{$\begin{array}{l}\text { Orientación decisión: individual (aunque con } \\
\text { mecanismos de flujo) } \\
\text { Posible preocupación presupuestaria: solo } \\
\text { respecto de la misma institución } \\
\text { Efectos presupuestarios: propios, en } \\
\text { defensoría, policías, tribunales y gendarmería. }\end{array}$} \\
\hline \multicolumn{2}{|c|}{$\begin{array}{l}\text { Jueces: decisión } \\
\text { individual de } \\
\text { sanción y cuantía }\end{array}$} & \multicolumn{2}{|c|}{$\begin{array}{l}\text { Orientación decisión: naturalmente individual } \\
\text { Posible preocupación presupuestaria: solo } \\
\text { respecto de la misma institución } \\
\text { Efectos presupuestarios: propios y } \\
\text { Gendarmería. Dependiendo de eficiencia } \\
\text { también en Ministerio Público y Defensoría }\end{array}$} \\
\hline \multicolumn{2}{|c|}{$\begin{array}{l}\text { Comisiones } \\
\text { de libertad } \\
\text { condicional }\end{array}$} & $\begin{array}{l}\text { Tribunales } \\
\text { superiores } \\
\text { recurso de } \\
\text { amparo }\end{array}$ & $\begin{array}{l}\text { Ministerio } \\
\text { de Justicia y } \\
\text { Presidente de la } \\
\text { República } \\
\text { Indultos }\end{array}$ \\
\hline \multicolumn{2}{|c|}{$\begin{array}{l}\text { Orientación decisión } \\
\text { (Chile): individual } \\
\text { Posible preocupación } \\
\text { presupuestaria: no. } \\
\text { Decisiones impactan } \\
\text { gendarmería }\end{array}$} & $\begin{array}{l}\text { Ministerio de Justicia } \\
\text { y Presidente de la } \\
\text { República } \\
\text { Indultos } \\
\text { Posible preocupación } \\
\text { presupuestaria: si } \\
\text { Decisiones impactan } \\
\text { presupuesto propio }\end{array}$ & $\begin{array}{l}\text { Orientación decisión } \\
\text { (Chile): } \\
\text { Consideración } \\
\text { naturalmente } \\
\text { individual } \\
\text { Posible preocupación } \\
\text { presupuestaria: no } \\
\text { Decisiones impactan } \\
\text { gendarmería }\end{array}$ \\
\hline
\end{tabular}

La Figura 3 -centrada en el caso ilustrativo de la prisión, pero aplicable mutatis mutandi a otras clases de sanción- identifica a los distintos organismos que toman decisiones que contribuyen a la producción del output global del sistema penal. Ella muestra como las decisiones de los que se encuentran más arriba en la cadena dinámica afectan no solo a las decisiones de los organismos que se encuentran más abajo, sino que también tienden a impactar en los presupuestos de estos últimos. 
En particular, la Figura 3 pretende ilustrar cuatro fenómenos, más allá de la dispersión de tomadores de decisión, que contribuyen a la falta de coordinación en la producción del resultado global y a la falta de responsabilidad a su respecto.

En primer lugar, la Figura 3 ilustra que los tomadores de decisión ubicados al inicio de la cadena -con excepción de la legislación, que sin embargo funciona sin control presupuestario en materia penal en Chile- en general imponen (en vez de asumir) cargas y los tomadores de decisión que se encuentran más abajo tienden a ser más bien organismos que absorben los efectos presupuestarios de decisiones de otros. Con ello, no hay presiones presupuestarias internas que controlen naturalmente el resultado del sistema ${ }^{34}$.

En segundo lugar, los criterios de toma de decisión están configurados en casi todos los casos -con la excepción de la no muy responsable legislación y del ejecutivo en materia de indultos- con orientación individual ${ }^{35}$. Los fiscales persiguen, o no, casos en base al mérito (pruebas, cuantía, etc.). Los jueces naturalmente condenan o absuelven, y fijan la pena, en base al mérito individual. Y, en algo que es tal vez idiosincrático, los tomadores de decisión a nivel de libertad condicional también asumen esa lógica. Ello implica que, más allá de preocupación por la propia eficiencia de sus procesos (sacar la mayor cantidad de condenas o tramitaciones posibles), no hay preocupación por los resultados del sistema ${ }^{36}$.

En tercer lugar, las medidas que integran cada estadio no se establecen con la misma lógica. El quantum global del primero estadio -el número total de delitos conocidos- sigue dinámicas que poco tienen que ver con la administración. La capacidad de procesamiento de las policías y del Ministerio Público depende de su propia disponibilidad de recursos y de la eficiencia de su uso. Y la producción del resultado (individual y global) sanción depende de interacciones estratégicas con los defensores, de la capacidad de procesamiento de esas interacciones por tribunales y de su valoración como conducente, o no, a sanciones ${ }^{37}$.

En cuarto lugar, aunque el paso de un estadio a otro se ve fijado naturalmente con una misma unidad cuando se considera a nivel individual -el mismo delito es conocido, perseguido, sometido a proceso y sancionado al pasar de estadio en estadio- a nivel de campo no hay correspondencia entre la demanda que supone cada estadio y la oferta de procesamiento del siguiente.

Esto es: la policía no cuenta con recursos para procesar la demanda de investigación que supone cada delito del que tiene conocimiento, el Ministerio Público no puede procesar cada caso, y el sistema de sanciones no tiene capacidad de absorber todas las demandas de sanción requeridas por el sistema judicial, etc. Por lo mismo, el resultado global depende

\footnotetext{
34 Aunque referido al caso institucionalmente más complejo de los Estados Unidos, así también BARKOW (2017) pp. 43-44; ZimRING y HaWKINS (1993) pp. 211-15.

35 STUNTZ (1997) p. 4.

36 Informalmente, el ejecutivo a través de la Dirección de Presupuestos, del Ministerio de Justicia al negociar su presupuesto y de decisiones de indulto, podría asumir una función de esta clase. Conocer exactamente, en los hechos, cómo ello opera, es un trabajo de investigación en sí mismo que me interesa desarrollar. Pero, en cualquier caso, ello solo impactaría en poder identificar posibilidades de modelación del control a partir de estructuras de funcionamiento ya establecidas. Un modelo informal de esta clase ofrece desventajas naturales, porque no puede incidir sobre el output de tomadores de decisión orientados individualmente.

37 STUNTZ (1997) p. 4.
} 
en una medida mucho mayor de la oferta de procesamiento disponible en cada estadio que de, por una parte, la demanda total (considerada en cada estadio) o, por la otra, de una consideración racional de resultados globales buscados.

Varias consecuencias se siguen de lo anterior. En primer lugar, el sistema de justicia penal tiene una escasez material permanente frente a la demanda formal de acción que se le presenta. En efecto, mientras sus necesidades de acción son fijadas formalmente en relación con cada delito -el Estado de derecho pretende establecer la necesidad de respuesta frente a cada transgresión relevante-, materialmente considerado, en realidad su capacidad de acción depende de disponibilidad, coordinación y eficiencia en el uso de sus recursos ${ }^{38}$. En segundo lugar, el hecho de que sean los propios recursos y modos de acción de la administración aquello que establece el quantum total de sanciones, implica que el nivel agregado de delitos tiene poca influencia (directa) sobre el output total del sistema. El castigo como fenómeno agregado es, en general, una variable influenciada por la organización de la administración; no, en cambio, por las tasas de delincuencia ${ }^{39}$.

Por ahora, ello permite asumir una tensión que es a la vez fuente de problemas y oportunidades en el funcionamiento del sistema penal: no hay organización de campo tendiente a controlar resultados, pero, al mismo tiempo, como el output global tiende a depender de variables más o menos controlables por los organismos (sus recursos y la eficiencia en su actuar), ello presenta alguna posibilidad de contrapesar el desorden natural del sistema. Ello, sin embargo, en la medida en que haya orientación a su uso con esa pretensión. Como veremos, en el diseño institucional actual ese, desafortunadamente, no es el caso.

\subsection{LA IMPROBABILIDAD DEL REALINEAMIENTO ESPONTÁNEO}

La estructura orgánica referida en la sección anterior permite explicar la improbabilidad del re-alineamiento espontáneo del sistema penal. Si uno mira la misma información sobre la dispersión y características del sistema de tomas de decisión, pero se concentra ahora en mirar quiénes influyen sobre la producción de los factores centrales "ingresos" y "egresos", esto es fácil de observar.

En particular, los organismos que contribuyen a su producción son distintos y no tienden a coordinarse en relación con los resultados que producen. En el ámbito de los ingresos, el número de prisiones preventivas depende, en lo esencial, de decisiones del Ministerio Público. El número de condenas conducentes a penas efectivas depende de la interacción del Ministerio Público, las defensas y los tribunales -y en buena medida de la disponibilidad de espacio para procesamiento de los tribunales-. Pero todos estos organismos toman decisiones en base a consideraciones individuales y, cuando tienen una orientación

\footnotetext{
38 Entre nosotros, este hecho se encuentra en la base de la crítica a la pretensión de definición estricta de legalidad del Ministerio Público (obligación de perseguir todo) y la generación excepcional de oportunidad.

39 Aquí es importante la calificación "directa” en la afirmación "el delito no tiene influencia directa en el quantum del castigo”. Aumentos en las tasas de delincuencias (o en su percepción) sí pueden tener influencia indirecta, por la vía de impulsar al sistema político a aumentar la disponibilidad de recursos o los mecanismos de acción conducentes a producir castigo institucional. Véase LACEY et al. (2018); Pfaff (2014, 2017).
} 
"gerencialista" referida al resultado global, ella tiende simplemente a impulsar la producción de un mayor número de ingresos ${ }^{40}$.

En materia de egreso, las salidas dependen de decisiones de determinación de la pena (tomadas en base a otras consideraciones al momento de la condena) y de su interacción con mecanismos de salida anticipada. Las salidas anticipadas de la cárcel (ante todo: libertad condicional) dependen de las comisiones de libertad condicional y de los tribunales superiores de justicia (vía recurso de amparo). Aquí también las decisiones tienden a integrar el puro punto de vista individual. Y, en lo que puede verse, los organismos no interactúan entre sí para intentar equilibrar la ecuación conducente al resultado final.

Uno puede, sin embargo, invertir la mirada y pasar a considerar que el output total de cada uno de los mecanismos que influye en la ecuación referida en la Figura 3 son "instrumentos" para realinear los resultados. En un sistema institucionalmente centralizado como el chileno ${ }^{41}$, producir ese efecto es formalmente sencillo. Ejemplo: bastaría que alguien con competencia en el sistema (y con preocupación por sus resultados) vea que entre $\mathrm{N} 1$ y N2 se produjeron desajustes en el nivel de ingresos (o tiempos de cárcel demasiado largos y, con ello, menos oportunidades para el egreso, o cualquier otro factor relevante) y que tenga información suficiente sobre dónde tuvieron lugar los desajustes, para que pueda establecerse un modo de acción conducente a realinear el sistema en la producción de encarcelamiento. Como hemos visto, parte relevante del problema en Chile es que no hay organismo alguno que tenga esa función.

La cuestión se torna más compleja, en cambio, si el resultado que se quiere controlar no es solo el nivel de encarcelamiento, sino el nivel total de castigo o el impacto social del castigo. Para pasar a considerar el nivel total de castigo, basta con ampliar los "ingresos" a toda medida que tenga peso aflictivo, incluyendo otras medidas cautelares, multas (aunque a diferencia de las otras sanciones ellas no representan una carga fiscal), penas sustitutivas, y libertades condicionales en ejecución. Al igual que en el caso del encarcelamiento, basta mantener estable el nivel de "ingresos" y "egresos" a nivel agregado, o respecto de un tipo de medidas en particular, para que los resultados se estabilicen; y es teóricamente posible también orientar una disminución. Pero como no se trata de sanciones definidas del mismo modo, el juego con el nivel de un tipo de medidas puede producir efectos en otras.

Ejemplo: las penas sustitutivas implican (al menos teóricamente) un aumento en niveles de sanción, pero ellas son al mismo tiempo medidas de evitación de la cárcel. Formalmente visto, disminuir el umbral desde el que se imponen penas sustitutivas (y no así cárcel efectiva) puede ser una medida eficiente para disminuir los ingresos a la cárcel. Pero ella no disminuye formalmente el nivel total de castigo. $\mathrm{Y}$, materialmente visto, una medida de esta clase puede incluso ampliar el nivel de castigo: las penas sustitutivas son más baratas

${ }^{40}$ En los Estados Unidos, varios autores le atribuyen a este punto -y al mejoramiento de los sistemas de gestión por avance tecnológico- la mayor parte de la responsabilidad por la explosión carcelaria. Así, por ejemplo, Pfaff (2017) cap. 5; Stuntz (2006, 2010).

${ }^{41}$ El contraste es con Estados Unidos, donde la fragmentación y complejidad institucional hace mucho más difícil controlar los resultados a nivel central. 
de imponer y, bien diseñadas, pueden proveer de niveles adecuados de control, con lo que pueden ser una herramienta eficiente de expansión antes que de retracción del castigo ${ }^{42}$.

Como los regímenes sancionatorios son difícilmente conmensurables entre sí -técnicamente expresado: pueden ser medidos a nivel ordinal (¡cuál es más gravoso?) pero no a nivel de intervalo- y tienen impacto cruzado, la optimización de su uso requiere ser medida de otro modo. En situación de conocimiento perfecto, el nivel óptimo de uso debiera ser establecido midiendo la combinación, alcanzable con los recursos disponibles, con mejor rendimiento social y que además se justifique frente a otras alternativas de usos de recursos públicos -el modelo básico de evaluación costo beneficio ${ }^{43}$. Ello implicaría considerar no solo el efecto directo de las sanciones, sino también efectos colaterales en la vida futura del condenado- y con ello la tendencia a la generación de reincidencia -y en sus cercanos- y con ello la tendencia a la generación de más delincuencia.

Uno puede dudar probablemente con razón que ese óptimo pueda ser conocido del todo. Varios de los factores involucrados dependen de fijaciones políticas cuya corrección no es fácil de medir y los efectos de un sistema sancionatorio son conocidamente problemáticos de evaluar ${ }^{44}$. Pero lo que al menos debe pedirse a un sistema de justicia penal, es que opere con expectativas claras en relación con los resultados que debe producir y que se produzca coordinación para intentar mantenerse en esas metas. La próxima sección identifica distintas modalidades en que ello puede tener lugar.

\section{MODELOS DE CONTROL ADMINISTRATIVO DEL RESULTADO DEL SISTEMA PENAL}

Como vimos, el resultado agregado del sistema depende de la relación entre varios actos institucionales que producen ingresos y egresos. Si esos números pudieran ser manipulados de modo tal de no producir déficit, el control de los resultados del sistema sería sencillo. La misma homonimia con el lenguaje contable da cuenta de lo frecuente que es el control de procesos de equilibrio de ingresos y egresos: toda organización de la economía formal $-\mathrm{y}$ no solo en el ámbito público- tiene que controlar que sus egresos no sobrepasen irracionalmente sus ingresos. Aquí, por cierto, los "ingresos" (de presos) son los que producen egresos presupuestarios (gastos) y, al revés, los "egresos" (de presos) se corresponden con disminuciones de gastos. Por lo que, si la administración pensara como empresa, debiera naturalmente buscar maximizar los egresos (de presos) y disminuir los ingresos.

Para poder dar cuenta de los desafíos que un sistema administrativo del sistema de justicia penal enfrenta, la primera cuestión relevante es entender por qué el sistema no puede simplemente asumir esa lógica contable. En otras palabras, por qué la Figura 3 tiene la estructura, aparentemente irracional, que exhibe. Esa es la misión de la primera sección de

42 En la literatura comparada, esto se conoce como el debate del ensanchamiento de la red de castigo (netwidening). Véase CoHEN (1985). Sobre la expansión de las penas sustitutivas a niveles masivos y la necesidad de considerar su extensión en el control de la magnitud global del castigo, PHeLps (2017).

43 BarKow (2017) pp. 43-44; LA MiSMA (2019) pp. 180-81.

44 Véase a este respecto la revisión de la literatura sobre el efecto general de disuasión de variaciones en los regímenes de castigo en Webster y Dоов (2012). 
Wilenmann, Javier “ "Controlando los resultados del sistema penal sobre el derecho administrativo del derecho penal"

este apartado: explicar qué aspectos esenciales a la identidad del sistema penal impiden una solución administrativa sencilla. Las demás secciones, asumiendo este diagnóstico, presentan los distintos modelos que pueden, pese a ello, permitir un control de esta clase.

\subsection{El “PROBlema” Básico: El PRINCIPIO DE CUlPABILIDAD, LA RACIONALIDAD DE LA CULTURA PENAL Y EL RESULTADO GLOBAL}

El lector, en particular perteneciente al ámbito legal o a la filosofía, puede preguntarse a partir de las frías descripciones de los apartados anteriores: ¿y dónde está la persona individual? ¿Dónde está el mérito individual de la sanción? ¿Dónde está el sufrimiento de la víctima del delito y del condenado? ¿Dónde están las nociones de responsabilidad que permean al derecho penal?

Hasta ahora, la descripción muestra un sistema desordenado y sin preocupación por consideraciones globales, como si ello fuera el resultado simplemente de un mal diseño y no, en cambio, de arreglos institucionales que responden a ideas que tienen buena razón de ser. La adición de la cultura legal en materia penal y el diseño institucional del sistema tal vez puedan ser vistos como causantes de la configuración desordenada de la producción del output total del campo. ¿ Sugiere esto acaso que esa configuración cultural -y el diseño que la soporta- es sencillamente irracional?

Por supuesto, leer de este modo esta contribución sería un error. La programación del sistema en base a tomas de decisión con orientación al caso particular responde a las ideas que los penalistas, con buenas razones, estamos acostumbrados a defender: la pena se justifica en la medida en que un proceso de imputación de responsabilidad individual por realizar un delito justifique su imposición. Aunque dado el enorme volumen de causas que la burocracia tiene que manejar, mantener esa orientación individual en todos los casos es imposible ${ }^{45}$, al menos en la aplicación de sanciones considerables hay buenas razones para que, al menos ciertos actores, mantengan una configuración como tomadores de decisión independientes. Ese es el desafío administrativo global del sistema penal: generar orientación a la racionalización de resultados globales en un campo de tomas de decisión naturalmente orientado al caso individual.

El desafío implica que ciertas opciones, que podrían estar abiertas en otros sistemas burocráticos, simplemente no entren en consideración. En particular, el tomador de la decisión directa respecto de los ingresos -los tribunales penales- simplemente no puede asumir una lógica colectiva de administración. Una pregunta distinta es por la posición del otro actor central a nivel de ingresos, los fiscales. En particular, en la medida en que su configuración, como en Chile o Estados Unidos, sea a la producción del mayor número de sanciones posibles y de la mayor entidad posible, el control que tienen sobre buena parte del sistema es un problema ${ }^{46}$. Pero ello sugiere que, a nivel global, el Ministerio Público debiera participar de los arreglos que permitan controlar los resultados del sistema en cierta clase de casos, además de asumir la naturaleza administrativa global de su empresa. Sobre

\footnotetext{
45 Sobre las diferencias respecto de distintas clases de delitos, véase NATAPOFF (2017) pp. 71-77.

46 Barkow (2019) cap. 8; Pfaff (2017a) cap. 5; Pfaff (2017b); Sklansky (2018).
} 
ello volveremos más adelante. En cualquier caso, en casos en que haya decisión de persecución, la perspectiva tiene que ser naturalmente individual.

El problema del caso chileno se encuentra, sin embargo, no solo en la configuración del programa de acción del Ministerio Público, sino en la falta de actores que asuma del todo una orientación naturalmente sistemática. El actor tradicional que en teoría penológica asume ese punto de vista - los tomadores de decisión de libertad condicional- no lo hace, ya que en Chile ha pasado a imperar la consideración de que se trata de un instrumento de orientación puramente individual. La única excepción, por ello, se encuentra Ministerio de Justicia en los casos en que decide hacer indultos masivos -algo improbable dados los costos políticos involucrados y que en el cualquier caso tiene la forma de solución parche- y en la legislación, en condiciones que los incentivos del sistema político tienden a no coincidir con preocupaciones de esta clase.

En lo que sigue, pretendo explorar los distintos modelos que existen para efectos de incorporar una perspectiva global en el funcionamiento del sistema. El análisis parte de una asunción básica: se requiere alguien que controle la racionalidad de los resultados globales. Es probable que un sistema de justicia penal que muestre tendencia al desalineamiento no pueda producir ese efecto sin que exista un organismo especialmente orientado a ello. El sistema político podría hacerlo, pero una vez que hay desalineamiento, el costo político de manipular por puras consideraciones orgánicas los resultados del sistema es demasiado alto frente a su eventual beneficio, a saber, mostrar responsabilidad fiscal y por la producción de resultados positivos de largo plazo ${ }^{47}$.

En el caso de la burocracia, los actores centrales del sistema no asumen una orientación natural de este tipo. Las razones son varias: los actores no tienen incentivos naturales a considerar el impacto global de sus acciones - por que las organizaciones de las que participan no necesitan internalizar ese costo-, los actores no cuentan con información sobre ese impacto, y ella es, en cualquier caso, compleja y cambiante. Como la información cambia constantemente y depende de muchos organismos, ella solo puede obtenerse si -a la forma de un banco central- hay una burocracia (en este caso ciertamente mucho más pequeña) encargada de recolectar y procesarla constantemente. Y, al igual que un banco central, ese organismo puede tener modos directos e indirectos que tiendan a equilibrar la ecuación y evitar la producción de inflación.

En general, en lo que dice relación con el funcionamiento del sistema penal, uno puede así distinguir binariamente dos modelos de agencia que tenga una función de esta clase: una agencia que cuente con mecanismos directos de alienación -bajo el modelo de la sentencing commission de Minnesota o bajo el modelo de comisiones de libertad condicional con información centralizada y orientación a la alineación- y un modelo que solo cuente con mecanismos indirectos de alineación, esto es, que solo pueda (intentar) imponer consideraciones sistemáticas en otros actores. Un sistema correctamente diseñado debiera tender a ambas soluciones. El organismo global no puede actuar sin orientación parcialmente sistemática de los distintos componentes del sistema, y es improbable que esa orientación se

47 BARKOW (2005) p. 716; LA MISMA (2019) pp. 110-12. 
WilenMann, Javier " "Controlando los resultados del sistema penal sobre el derecho administrativo del derecho penal"

genere del todo o, al menos, produzca los resultados que debe producir, sin que exista un organismo global de esta clase. En lo que sigue, describiré brevemente los dos modelos.

\subsection{LA SOLUCIÓN ORGÁNICA GLOBAL CON INCIDENCIA DIRECTA}

Una agencia global con competencia directa para producir la alineación del sistema se define por contar con instrumentos directos para realizarlo. En lo que alcanzo a ver, hay dos modos comparados puros de esta clase: hacerlo sobre la base del control de la determinación de la pena tanto a nivel legislativo como judicial, por una parte, y hacerlo sobre la base de centralización en la toma de decisiones de libertad condicional.

El primer modelo fue popularizado por la exitosa experiencia del Estado de Minnesota ${ }^{48}$ y luego se generalizó en distintas comisiones de sentenciamiento en varios Estados de la unión -con variaciones relevantes en su diseño institucional ${ }^{49}$. La idea del modelo básico es sencilla: si la población carcelaria depende exclusivamente de la relación entre ingresos y egresos por cumplimiento (o muerte), entonces manipular centralizadamente los tiempos de cumplimiento permite mantener equilibrada la ecuación (o, si se fijara esa meta, disminuirla).

En el modelo de Minnesota, la libertad condicional fue así eliminada, ya que ella generaba trato desigual, ${ }^{50}$ distorsiones y aumento de complejidad. Sin libertad condicional, la información sobre la composición actual de la población carcelaria y las expectativas de flujos de ingresos sería suficiente para poder equilibrar la ecuación en caso de variaciones en los factores ${ }^{51}$. Entregando competencias de recolección de esta información, parte de la competencia legislativa en la fijación de la cuantía de las penas fue también transferida a la comisión de determinación de la pena: el sistema político pasó a solo fijar gravedad en una escala ordinal, pero el quantum asociado a cada punto de la escala es fijado por la comisión considerando, entre otras cosas, objetivos respecto a la población carcelaria total. Al introducir nuevos delitos, hacer variar la gravedad de delitos o manipular otras partes del sistema, el legislador debía además contar con informes de la comisión ${ }^{52}$. Todo ello bajo la idea de que tanto la implementación de las directrices de determinación de la pena, como todas las modificaciones posteriores, debiera orientarse a que la población carcelaria no sobrepase el $95 \%$ de la capacidad del sistema ${ }^{53}$.

El modelo exactamente contrario se fija no en las decisiones que influyen sobre determinación de la pena, sino en las decisiones que tienen lugar después de la ejecución -en particular el otorgamiento de libertad condicional. Al menos en teoría, en un sistema que fije amplias oportunidades de la administración para otorgar libertad condicional- por

\footnotetext{
48 Sobre la historia de su implementación, el éxito en la resistencia inicial a presiones políticas y sus problemas ulteriores, véase BARKOW (2005) pp. 773-77; FrASE (2005a) pp. 139-59.

49 Una visión general es ofrecida por Frase (2005b) pp. 1194-1206.

50 Esta es la crítica original: falta de capacidad de manejar la producción de resultados similares.

51 Frase (2005b) pp. 1198s.

52 Manteniendo la comparación con un banco central, uno podría pensar en instrumentos todavía más agresivos de control de la ecuación. Por ejemplo, la comisión podría no fijar la cuantía solo al momento de la determinación, sino manipular tasas de disminución del tiempo de condena de modo tal de producir el efecto buscado.

53 Frase (2005a) pp. 146s.
} 
ejemplo, porque funciona solo imponiendo un tiempo máximo de privación de libertad, pero dejando desde un momento temprano disponible la posibilidad de otorgar libertad condicional (sentenciamiento indeterminado) ${ }^{54}$-, la administración tiene una capacidad casi completa de manejar los resultados globales de encarcelamiento. Si ello además se controla a nivel centralizado por una agencia con información global amplia, la administración podría teóricamente controlar directamente que los resultados del sistema se alineen ${ }^{55}$.

Por supuesto, nada impide que los dos modelos se combinen. Una agencia podría tener tanto control centralizado de los resultados de libertad condicional, como influencia sobre los estándares de determinación de la pena y, además, forzar al legislador a sincerar los costos de la política criminal. Eso le permitiría además más libertad para balancear la ecuación (o generar disminución de la población carcelaria dirigida a un objetivo específico) sin recargar un solo tipo de instrumento.

Aunque un modelo de acción directa sobre el sistema tiene beneficios evidentes, se encuentra sujeto a varios problemas. Los más obvios se refieren a su posible falta de viabilidad política ${ }^{56}$. Es poco probable que, en nuestras condiciones, el sistema político quiera delegar tantas competencias como en el modelo de Minnesota (más allá de la pregunta constitucional no planteada en Chile sobre los límites de esa delegación, al menos en la cuantificación de la gravedad de los delitos) o en el modelo del sentenciamiento indeterminado. También es poco probable que, como un banco central, el organismo en cuestión se encuentre suficientemente aislado frente a la obvia hostilidad política que tendría ${ }^{57}$. Además, en el caso del manejo de la libertad condicional, su uso eficiente -en base a diagnósticos y planes de reinserción y pronósticos de reincidencia- requiere de un aparato burocrático mucho más extenso y especializado que el que existe en Chile. El modelo combina aquí expertise si se quiere económica global en manejar resultados globales -algo seguramente no muy difícil de implementar- y expertise individual psicológica y de acompañamiento social en el manejo y diagnóstico de casos individuales -algo que requiere inversiones masivas y capital humano que probablemente requiera mucho tiempo para su desarrollo.

Aunque teóricamente llamativa, las dificultades de implementación de una burocracia que administre eficientemente las plazas de cárcel combinando información de sentenciamiento y pronósticos respecto de los condenados, hace poco probable que en un país como el nuestro pueda desarrollarse.

\footnotetext{
54 Sobre el modelo en cuestión véase TONRY (2013).

55 Sobre los intentos anteriores a las reformas de los años 1970 de operar de este modo en algunos Estados de los Estados Unidos, véase BARKOW (2005) pp. 740s.

56 En la práctica, ello ha sido determinante. El fracaso más relevante se refiere a la comisión de sentenciamiento federal en los Estados Unidos. Aunque fue diseñada, bajo el modelo de Minnesota, para entre otras cosas controlar los resultados del sistema, no tuvo capacidad de aislamiento político y tendió más bien a producir los efectos contrarios. Véase BARKOW (2005) pp. 765-66, LA MISMA (2019) pp. 170s.

57 BARKOW (2019) cap. 8.
} 
WilenMann, Javier " "Controlando los resultados del sistema penal sobre el derecho administrativo del derecho penal"

\subsection{LA AGENCIA GLOBAL SIN COMPETENCIA DIRECTA: INTRODUCIENDO ORIENTACIONES SISTEMÁTICAS, Y COORDINACIÓN GLOBAL, EN LOS ORGANISMOS TOMADORES DE DECISIÓN}

Un modelo probablemente más sencillo de implementar y que no exhibe los mismos riesgos políticos de una agencia con poder directo, se caracteriza por no hacer uso de mecanismos directos de incidencia sobre el output del sistema, sino más bien por actuar para imponer que los organismos con competencia directa tengan una orientación global de este tipo y preocupación por los resultados. Al mismo tiempo, la falta de mecanismos directos de acción genera riesgos de impotencia.

La idea aquí también es sencilla. Si un organismo cuenta con amplia información de ingresos y egresos, puede determinar dónde se han producido variaciones que han generado, o podrían generar, inflación carcelaria. Si se le impone un mandato externo de producción de un resultado específico (por ejemplo: solo tener población carcelaria hasta el $95 \%$ de la capacidad del sistema), el organismo puede ver qué cambios en el uso de los "instrumentos" (mecanismos de filtro de ingreso o mecanismos de egreso) podrían producir ese resultado. Y, en ese caso, el organismo puede intentar imponer a los actores del sistema la adopción de estas metas.

El caso obvio en el que la agencia puede hacer esto dice relación con la legislación: una agencia que maneje información global puede informar sobre impactos esperados de modificaciones al sistema o actuar como experto en la determinación de las medidas necesarias para alcanzar un resultado determinado ${ }^{58}$. Ello al menos ayudaría a hacer visibles los costos del sistema y de las decisiones político-criminales ${ }^{59}$.

Sin embargo, nada impide como cuestión de diseño que un organismo de esta clase pueda también generar metas respecto del Ministerio Público en el uso de salidas alternativas y en la solicitud de penas; a los tribunales en las prácticas de determinación de la pena, y respecto de los actores que generan salidas anticipadas.

El problema central de un sistema de esta clase dice relación con la falta de capacidad de imponer las metas. Por diseño democrático básico, la imposición de metas al legislador es prácticamente imposible -el sistema político solo se vería forzado a sincerar $\operatorname{costos}^{60}$. Respecto del resto de los organismos a los que podría coordinar e intentar imponer la asunción de una orientación al resultado global, la pura fijación de metas sin consecuencias puede ser perfectamente inútil. El principal desafío, por ello, es pensar cómo puede generarse el efecto de imposición.

El mecanismo de forzamiento más intenso que alcanzo a reconocer es económico: manejo de presupuesto. Esto es, un organismo de esta clase -de estar suficientemente aislado políticamente y, pese a ello, coordinado con la dirección de presupuestos- podría fijar

\footnotetext{
58 Esto es definido como la función básica de un organismo de esta clase: Frase (2005b) p. 1198.

59 En todos estos casos, el organismo puede tener métodos más o menos sofisticados para generar metas. El mínimo dice relación a la pura orientación a producir alineación (o deflación) sancionatoria. En ese caso, basta manipular la ecuación de un modo determinado. Ello puede complementarse con modelos de análisis costo beneficio más o menos complejos.

${ }^{60}$ Pese a ello, con buen diseño institucional el caso más exitoso -Carolina del Norte- ha funcionado simplemente por la vía de obligar a esto. BARKOW (2005) pp. 781-86.
} 
metas de acción cuyo incumplimiento (en rangos relevantes) implique costos presupuestarios. $\mathrm{O}$, al revés, que el cumplimiento de las metas pueda ser traspasado a la institución y sus agentes.

Ello, por cierto, se enfrenta a dificultades relevantes. En programas no esenciales del Estado, imponer costos por no cumplir metas es políticamente más viable, porque la parálisis del programa es soportable. Pero tratándose de instituciones esenciales al funcionamiento del Estado, utilizar la reducción presupuestaria como mecanismo de internalización de los malos costos de decisión también externaliza el costo del incumplimiento en terceros (los presos, los imputados, etc.). Y la utilización de mecanismos de incentivo, si bien está exenta de ese peligro, siempre puede contribuir a distorsiones por producir brutamente los resultados.

En cualquier caso, el mínimo al que un sistema desalineado pero que se asume como responsable debe tender, es a producir una agencia que actúa como grupo de interés, con miembros con influencia en todos los organismos del sistema, que busque al menos generar racionalización cuantitativa del output del sistema.

\section{CONCLUSIONES}

Al tratarse de un sistema burocrático con un enorme costo fiscal e impacto social, el control de los resultados globales del sistema penal debiera ser una preocupación central tanto de la legislación como de la burocracia. Las características del campo hacen improbable que el sistema se alinee ya en la pura producción de resultados ordenados. Y, una vez que se desalinea como tiene lugar en Chile y en las Américas (con la excepción de Canadá), es aún más improbable que pueda equilibrarse sin conducción central.

Imponer orientación al control de los resultados globales del sistema es, ciertamente, un desafío administrativo mayor. Como campo de tomadores de decisión que miran al caso individual y no se coordinan entre sí, esa orientación no es natural al sistema. Y, sin embargo, no imponerla constituye una irresponsabilidad fiscal, con la seguridad pública y con los afectados por el sistema.

Más allá de su relevancia científica en el análisis de las dinámicas del sistema penal, al explicar las razones por las que se necesita un organismo que controle la generación de esa orientación, y analizar algunos modelos que pueden servir a ello, este artículo espero pueda generar una contribución al menos inicial a generar sensibilidad en el mundo legal y político sobre el tema.

\section{BIBLIOGRAFÍA CITADA}

Ariza, Libardo José; e Iturralde, Manuel (2018): "Transformations of the Crime Control Field in Colombia", en Carrington et al. (eds.), The Palgrave Handbook of Criminology and the Global South (Cham, Palgrave Macmillan) pp. 687-708.

Aviram, Hadar (2017): “Book Review: Locked in: 'The True Causes of Massive Incarceration and How to Achieve Real Reform' and 'Breaking the Pendulum. The Long Struggle over Criminal Justice”, Law \& Society Review, vol. 51, No 4: pp. 1017-23. 
WiLenMann, Javier "Controlando los resultados del sistema penal sobre el derecho administrativo del derecho penal"

BARKOw, Rachel (2005): “Administering Crime”, UCLA Law Review, vol. 52: pp. 715-814. BARKow, Rachel 2014): "Criminal Law as Regulation”, New York University Journal of Law \& Liberty, vol. 8: pp. 316-42.

Barkow, Rachel (2017): “The Criminal Regulatory State”, en Dolovich, Sharon y NATAPOFf, Alexandra (eds.), The New Criminal Justice Thinking (Nueva York, New York University Press) pp. 33-52.

BARKow, Rachel (2019): Prisoners of Politics (Cambridge (MA), The Belknap Press of Harvard University Press).

Blumstein, Alfred, y Cohen, Jacqueline (1973): "A Theory of the Stability of Punishment", The Journal of Criminal Law \& Criminology, vol. 64, No 2: pp. 198-207.

ChIAO, Vincent (2017): "Mass Incarceration and the Theory of Punishment", Criminal Law and Philosophy, vol. 11, No 3: pp. 431-52.

CHIAO, Vincent (2018): Criminal Law in the Age of the Administrative State (Nueva York, Oxford University Press).

Cohen, Stanley (1985): Visions of Social Control (Glasgow, Polity Press).

DeShazo, Peter, y Vargas Juan Enrique (2006): Evaluación de La Reforma Judicial en América Latina (Santiago, CEJA).

DiMaggio, Paulo, y Powell, Walter W (1983): “The Iron Cage Revisited: Institutional Isomorphism and Collective Rationality in Organizational Fields", American Sociological Review, vol. 48, No 2: pp. 147-60.

Dolovich, Sharon, y Natapoff, Alexandra (2017): "Introduction: Mapping the New Criminal Justice Thinking”, en LAS MISMAS (eds.), The New Criminal Justice Thinking (Nueva York, New York University Press) pp. 1-29.

Doob, Anthony, y Webster, Cheryl Marie (2006): "Countering Punitiveness: Understanding Stability in Canada's Imprisonment Rates”, en Law \& Society Review, vol. 40, No 2: pp. 325-67.

Duce, Mauricio (2002): "Selección de casos en el nuevo Código Procesal Penal", Revista Chilena de Derecho, vol. 29, No 2: pp. 247-72.

Ewald, Alec, y UgGen, Christopher (2012): “The Collateral Effects of Imprisonment on Prisoners", en Petersilia, Joan, y Reitz, Kevin R (eds.), The Oxford Handbook of Sentencing and Corrections (Nueva York, Oxford University Press) pp. 83-103.

FonseCA, David S. (2018): "Expansion, Standardization, and Densification of the Criminal Justice Apparatus: Recent Developments in Brazil”, Punishment \& Society, vol. 20, No 3: pp. 329-50.

Frase, Richard (2005a): “Sentencing Guidelines in Minnesota, 1978-2003”, Crime \& Justice, vol. 32: pp. 131-219.

Frase, Richard (2005b): "State Sentencing Guidelines: Diversity, Consensus, and Unresolved Policy Issues”, Columbia Law Review, vol. 105: pp. 1190-1232.

Friedman, Lawrence (1975): The Legal System (Nueva York: Russell Sage Foundation).

Garland, David (2002): The Culture of Control (Oxford/Nueva York, Oxford University Press).

GonzÁlez, Claudio (2018): Gestión, Gerencialismo y Sistema Penal (Montevideo, BdeF). 
Hagan, John, y Dinovitzer, Ronit (1999): “The Collateral Consequences of Imprisonment for Children, Communities, and Prisoners", Crime \& Justice, vol. 26: pp. 122-62.

Haney, Craig (2012): “The Psychological Effects of Imprisonment”, en Petersilia, Joan, y ReITz, Kevin R (eds.), The Oxford Handbook of Sentencing and Corrections (Nueva York, Oxford University Press) pp. 584-605.

Hathazy, Paul (2016): "Punitivism with a Judicial Face: Criminal Justice Reformers's International and Regional Strategies and Penal State-Making in Argentina, Chile and Beyond", Kriminologisches Journal, vol. 48, No 4: pp- 294-310.

HöRnLE, Tatjana (2013): "Moderate and Non-Arbitrary Sentencing Without Guidelines: The German Experience", Law and Contemporary Problems, vol. 76, No 1: pp. 189-210.

Horvitz, María Inés, y López, Julián (2002): Derecho Procesal Penal Chileno Tomo I (Santiago, Editorial Jurídica de Chile).

Iturralde, Manuel (2010): "Democracies without Citizenship: Crime and Punishment in Latin America”, New Criminal Law Review, vol. 13, No 2: pp. 309-32.

LACEY, Nicola (2008): The Prisoner's Dilemma (Cambridge, Cambridge University Press).

Lacey, Nicola, Soskice, David, y Hope, David (2018): "Understanding the Determinants of Penal Policy: Crime, Culture, and Comparative Political Economy", Annual Review of Criminology, vol. 1: pp. 195-220.

Mayeux, Sara (2018): “The Idea of 'the Criminal Justice System”, American Journal of Criminal Law, vol. 45, No 1: pp. 55-93.

Natapoff, Alexandra (2017): “The Penal Pyramid", en Dolovich, Sharon y Natapoff, Alexandra (eds.), The New Criminal Justice Thinking (Nueva York, New York University Press) pp. 71-98.

Palacios, Daniel (2011): "La Reforma Procesal Penal en Chile: Nuevos Agentes, sus Trayectorias y la Reestructuración de un Campo", Revista Política, vol. 49, No 2: pp. 43-70.

Pfaff, John (2008): “The Empirics of Prison Growth", The Journal of Criminal Law and Criminology, vol. 98, No 2: pp. 547-620.

Pfaff, John (2014): "Escaping from the Standard Story: Why the Conventional Wisdom on Prison Growth Is Wrong, and Where We Can Go From Here", Federal Sentencing Reporter, vol. 26, No 4: pp. 265-70.

Pfaff, John (2017a): Locked In: The True Causes of Mass Incarceration - and How to Achieve Real Reform (Nueva York, Basic Books).

Pfaff, John (2017b): "Prosecutorial Guidelines", Reforming Criminal Justice, vol. 3: pp. 101-20.

Phelps, Michelle (2017): "Mass Probation: Toward a More Robust Theory of State Variation in Punishment", Punishment \& Society, vol. 19, No 1: pp. 53-73.

President's Commission of Law Enforcement and Administration of Justice (1967): The Challenge of Crime in a Free Society. Disponible en https://www.ncjrs.gov/pdffiles1/ nij/42.pdf

Riego, Cristián (2000): “Chile”, en Maier, Julio et al. (eds.), Las Reformas Procesales en Latinoamérica (Buenos Aires, Ad-Hoc) pp. 167-95.

Ruddell, Rick, y Fear, Noelle (2005): “The Stability of Punishment Hypothesis Revisited: A Comparative Analysis", International Journal of Comparative Criminology, vol. 5, No 1: pp. 1-28. 
Salinero, Sebastián (2012): “¿Por qué aumenta la población carcelaria en Chile?”, Revista Ius et Praxis, vol. 18, No 1: pp. 113-50.

SavelsberG, Joachim J. (1994): "Knowledge, Domination, and Criminal Punishment", American Journal of Sociology, vol. 99, No 4: pp. 911-43.

Sklansky, David (2018): “The Problems with Prosecutors”, Annual Review of Criminology, vol. 1: pp. 451-69.

Sozzo, Máximo (2016): "Democratization, Politics and Punishment in Argentina”, Punishment \& Society, vol. 18, No 3: pp. 301-24.

STUnTZ, William (1997): “The Uneasy Relationship Between Criminal Procedure and Criminal Justice", The Yale Law Journal, vol. 107: pp. 1-77.

Stuntz, William (2006) "The Political Constitution of Criminal Justice", Harvard Law Review, vol. 119, No 3: pp. 780-851.

Stuntz, William (2010): The Collapse of American Criminal Justice (Cambridge (MA), Harvard University Press).

TOnRY, Michael (2013): “Sentencing in America, 1975-2025”, Crime \& Justice, vol. 42, No 1: pp. 141-98.

Webster, Cheryl M. y Doob, Anthony N. (2012): "Searching for Sasquatch: Deterrence of Crime Through Sentence Severity”, en Petersilia, Joan, y Reitz, Kevin R (eds.), The Oxford Handbook of Sentencing and Corrections (Nueva York, Oxford University Press) pp. 173-95.

Wilenmann, Javier (2020): "Neoliberal Policies and State Modernization in Chilean Penal Evolution”, Punishment \& Society (en prensa).

Wilenmann, Javier, Del Fierro, Nicolás, Medina, Francisco, y Olivares, Esteban (2019): "La determinación de la pena en la Práctica Judicial Chilena", Politica Criminal, vol. 14, No 27: pp. 456-90.

Zedner, Lucia (2002): "Dangers of Dystopias in Penal Theory", Oxford Jounal of Legal Studies, vol. 22, No 2: pp. 341-66.

ZimRING, Franklin (2010): "The Scale of Imprisonment in the United States: Twentieth Century Patterns and Twenty-First Century Prospects", The Journal of Criminal Law and Criminology, vol. 100, No 3: pp. 1225-46.

Zimring, Franklin, y Hawkins, Gordon (1993): The Scale of Imprisonment. (Chicago/Londres, The University of Chicago Press). 